\title{
Detecção de vazamentos em redes de
} distribuição de água pela aplicação do Método Iterativo do Gradiente Hidráulico Alternativo (MIGHA) para calibrar vazões mediante modelagem computacional

\section{Leakage detection in water distribution networks by applying the Alternative Hydraulic Gradient Iterative Method (MIGHA) to calibrate flows through computational modeling}

\author{
Data de entrada: \\ 26/11/2019 \\ Data de aprovação: \\ 01/04/2020
}

Rafael Brito Aguiar de Araujo ${ }^{1 *}$ | Alessandro de Araújo Bezerra ${ }^{2}$ | Marco Aurélio Holanda de Castro ${ }^{1} \quad$ DOI: https://doi.org/10.36659/dae.2021.060 ORCID ID

Araujo RBA (i) https://orcid.org/0000-0002-6193-7564

Bezerra AA (iD https://orcid.org/0000-0002-9243-0731

Castro MAH (iD https://orcid.org/0000-0001-5134-7213

\section{Resumo}

A detecção de vazamentos em redes de abastecimento é de suma importância para combater o desperdício de água potável, assim como melhorar a eficiência dos sistemas de distribuição de água. Neste trabalho foi desenvolvida uma rotina, utilizando como base o Método do Gradiente Hidráulico Alternativo (MIGHA), para calibrar as vazões de uma rede e posteriormente utilizar os resultados da calibração para detectar possíveis pontos de vazamentos. Os resultados obtidos mostram que o método consegue identificar os nós e os trechos onde os vazamentos possam estar localizados. Em relação ao cálculo da localização dos vazamentos nos trechos, os resultados foram satisfatórios quando o vazamento não possuía influência de outros vazamentos próximos.

Palavras-chave: Calibração. Vazamentos. MIGHA.

\section{Abstract}

Detection of leaks in supply systems is of paramount importance to combat the waste of drinking water as well as to improve the efficiency of water distribution systems. In this work a routine was developed, based on the Alternative Hydraulic Gradient Method (MIGHA), to calibrate the flow of a network and later use the calibration results to detect possible leakage points. The results show that the method can identify the nodes and sections where the leaks may be located. Regarding the calculation of the location of the leaks in the sections, the results were satisfactory when the leak has no influence of other nearby leaks.

Keywords: Calibration. Leaks. MIGHA.

\footnotetext{
${ }^{1}$ Universidade Federal do Ceará - Campus Pici - Fortaleza - Ceará - Brasil.

${ }^{2}$ Universidade Federal do Piauí - Campus Ministro Petrônio Portela - Teresina - Piauí - Brasil.

* Autor correspondente: rafaelbritodmahotmail.com.
} 


\section{INTRODUÇÃO}

Para Tsutiya (2006) e Heller e Pádua (2010), as redes distribuição de água são a parte do sistema de abastecimento formada por tubulações e acessórios instalados em logradouros públicos com o objetivo de fornecer água potável em quantidade, qualidade, pressões adequadas e de forma contínua aos consumidores, sejam esses residenciais, comerciais, industriais ou de serviço.Normalmente, as redes de distribuição de água são dimensionadas e operadas considerando que estas funcionam de forma contínua, ou seja, 24 horas por dia, em regime permanente e uniforme.

Soares et al. (2004) consideram que não existem sistemas estanques, ou seja, toda a rede de distribuição de água já implantada apresenta alguma parcela de perda de água. As companhias de água e saneamento enfrentam sérios desafios no que diz respeito à detecção e ao reparo de vazamentos nas redes de abastecimento de água. De acordo com os dados do Sistema Nacional de Informações Sobre Saneamento (SNIS) de 2015, o Brasil perde cerca de $40 \%$ da água tratada com vazamentos, submedições nos hidrômetros e fraudes. A detecção de vazamentos hoje é realizada, em grande parte, por equipes especializadas das companhias de água e saneamento, utilizando equipamentos que se fundamentam em princípios acústicos para detectar os pontos de vazamento.

Atualmente, a utilização de métodos computacionais para projetar e simular o comportamento das redes de distribuição de água e demais projetos de engenharia é de suma importância. A calibração é um dos procedimentos experimentais bastante utilizado por meio de métodos computacionais. Calibrar consiste em estabelecer, sob condições especificadas, a relação entre os valores indicados por medições e valores estabelecidos como padrão.
Para simular, computacionalmente, de forma adequada uma rede de distribuição, é necessário que haja a calibração dos coeficientes da rede de distribuição. A calibração da rede visa adequar um modelo computacional para que este possa simular a rede estudada com base em dados observados da rede real.

Como forma de diminuir os gastos com manutenção e aumentar a eficiência dos processos normalmente utilizados para detectar pontos de vazamento não visíveis em redes, este artigo tem como objetivo utilizar o Método Iterativo do Gradiente Hidráulico Alternativo (MIGHA) para realizar a calibração das vazões de uma rede por meio de uma rotina computacional, com o objetivo de detectar possíveis pontos de vazamento em redes de abastacimento de água.

\subsection{Calibração da Vazão em Redes de Distribuição e Método Iterativo do Gradiente Hidráulico Alternativo - MIGHA}

Para Walski (1983), uma definição mais precisa para calibração de um modelo de distribuição de água consiste em um processo de dois passos, no qual o primeiro consiste na comparação de pressões e vazões calculadas com as observadas em condições de operação do sistema e, o segundo, no ajuste dos dados de entrada do modelo para que haja concordância entre o modelo calculado e o sistema observado.

Rocha, Castro e Araújo (2009) mostram que a função objetivo depende do número total de trechos, ou seja, no quadrado dos desvios dos gradientes hidráulicos, como é mostrado na Eq. 1.

$$
\mathrm{f}_{\mathrm{obj}}=\sum_{\mathrm{n}_{\mathrm{T}}}\left(\nabla \mathrm{H}_{\mathrm{O}}-\nabla \mathrm{H}_{\mathrm{C}}\right)^{2}
$$


Em que $\mathrm{n}_{\mathrm{T}}$ é o número total de trechos, $\nabla \mathrm{H}_{\mathrm{O}}$ é o gradiente hidráulico observado e $\nabla \mathrm{H}_{\mathrm{C}}$ é o gradiente hidráulico calculado.

Guo e Zhang (1994) apresentam O Método Iterativo do Gradiente Hidráulico (MIGH), baseado na calibração da transmissividade (ou da condutividade) hidráulica de aquíferos subterrâneos.

$\mathrm{Na}$ versão alternativa do método, Schuster e Araújo (2004), os autores propuseram a Eq. 2.

$$
\mathrm{T}_{\mathrm{j}}^{\mathrm{i}+1}=\mathrm{T}_{\mathrm{j}}^{\mathrm{i}} \frac{\left|\nabla \mathrm{H}_{\mathrm{C}_{\mathrm{j}}}^{\mathrm{i}}\right|}{\left|\nabla \mathrm{H}_{\mathrm{O}_{\mathrm{j}}}^{\mathrm{i}}\right|}
$$

Em que T é a transmissividade, i é o número da iteração, j é o índice da célula, $\nabla \mathrm{H}_{\mathrm{o}}$ é o gradiente hidráulico observado e $\nabla \mathrm{H}_{\mathrm{C}}$ é o gradiente hidráulico calculado.

A cada iteração i será calculado o ângulo $\theta$ formado entre os vetores dos gradientes hidráulicos observados e calculado em cada célula j, conforme mostrado na Eq. 3.

$\cos \theta=\frac{\nabla \mathrm{H}_{\mathrm{O}_{\mathrm{j}}} \nabla \mathrm{H}_{\mathrm{C}_{\mathrm{j}}}}{\left|\nabla \mathrm{H}_{\mathrm{O}_{\mathrm{j}}}\right|\left|\nabla \mathrm{H}_{\mathrm{C}_{\mathrm{j}}}\right|}$

A aplicação em redes de distribuição manteve o estabelecido por Schuster e Araújo (2004) quanto ao cálculo do ângulo $\theta$ e da função objetivo (Eq. 1), substituindo apenas a transmissividade pela vazão do trecho.

Para Bezerra (2018), em função da proporcionalidade existente entre a vazão e o gradiente hidráulico calculado pela equação de Darcy-Weisbach, ou fórmula universal, utilizada para o cálculo da perda de carga, a vazão é calibrada por meio da Eq. 4.

$$
Q_{j}^{i+1}=Q_{j}^{i}\left(\frac{\left|\nabla H_{\mathrm{C}_{j}}^{\mathrm{i}}\right|}{\left|\nabla \mathrm{H}_{\mathrm{O}_{\mathrm{j}}}^{\mathrm{i}}\right|}\right)^{-0.5}
$$

Para que haja a possibilidade de calcular o gradiente hidráulico observado em todos os trechos da rede, Rocha, Castro e Araújo (2009) consideram o uso de redes de distribuição, as quais foram chamadas de Rede Calculada e Rede Observada. No caso do uso de uma rede fictícia, os autores definiram, ainda, uma rede denominada Rede Gabarito como descrito a seguir:

- Rede Calculada: é a rede na qual serão realizados os cálculos hidráulicos e obtidos os resultados calculados. Parâmetros iniciais estimados são usados e, a cada iteração, com base no valor dos gradientes hidráulicos calculados obtidos nessa rede, esses parâmetros são alterados, gerando novos resultados, cada vez mais próximos dos medidos em campo. A Rede Calculada é a que possui os consumos estimados, os quais se deseja calibrar.

- Rede Observada: rede que apresenta os mesmos parâmetros iniciais da rede calculada, no entanto, possui pressões fixadas de acordo com os dados reais conhecidos.

- Rede Gabarito: rede fictícia na qual todos os parâmetros de entrada e resultados hidráulicos são conhecidos. Pode ser utilizada como base para conferir os resultados encontrados após o uso do método.

No caso de redes de distribuição, o ângulo $\theta$ só pode possuir os valores $0^{\circ}$ ou $180^{\circ}$, logo, com o uso do método não é permitido que o mesmo trecho apresente sentidos de fluxo opostos nas redes calculada e observada. O MIGHA aproxima os parâmetros hidráulicos das Redes Calculada e Observada a cada iteração, inclusive o sentido da vazão nos trechos, até o valor especificado para a função objetivo (Eq. 1) ser alcançado.

Se o sentido do fluxo da vazão de algum trecho for oposto ao fluxo da vazão do respectivo trecho da rede gabarito, ou seja, com um ângulo $\theta=$ 
$180^{\circ}$, adota-se o valor da vazão na iteração anterior da rede calculada.

O consumo nodal é calculado por meio do valor absoluto entre a diferença da vazão de saída e a vazão de entrada dos trechos adjacentes ao nó em questão, como mostra a Eq. 5.

$$
Q_{C}=\left|Q_{S}-Q_{E}\right|
$$

Em que $\mathrm{Q}_{c}(\mathrm{~L} / \mathrm{s})$ é a vazão consumida em um nó da rede, $Q_{s}(L / s)$ é a vazão de saída em um nó da rede por meio dos trechos adjacentes a ele e $Q_{E}(L / s)$ é a vazão de entrada do nó por meio dos trechos adjacentes a esse nó na rede de distribuição.

O processo consiste, primeiramente, em calcular os gradientes hidráulicos entre os trechos da rede calculada e a rede observada, estimando os consumos nodais da rede de forma arbitrária. Com os valores dos gradientes, aplica-se a Eq. 4 para calcular as novas vazões dos trechos da rede. Após o cálculo das novas vazões, obtém-se os novos consumos nodais por meio da Eq. 5. Com os valores dos novos consumos nodais, realiza-se uma nova simulação para obter os novos gradientes hidráulicos. O processo deve ser repetido 100 vezes, adotando-se a iteração que atingiu o menor valor da função objetivo.

\section{MATERIAIS E MÉTODOS}

\subsection{Calibração das Vazões e Cálculos dos Novos Consumos Nodais}

A metodologia adotada para calibração foi realizada com base na apresentada por Rocha, Castro e Araújo (2009). Utilizou-se o software EPANET2 para realizar as simulações da rede e calcular as pressões em regime de escoamento permanente e uniforme.

Com os valores dos gradientes hidráulicos das redes calculada e observada, deu-se início ao processo iterativo. Nos trechos nos quais a vazão alterou o sentido de escoamento do fluxo, adotou-se a vazão do respectivo trecho da rede observada ao invés do valor da iteração anterior da rede calculada.

O método foi aplicado na rede de distribuição malhada (Fig. 1) proposta por Walski (1983) e modificada por Gambale (2000). Os dados referentes aos trechos e nós desta rede são apresentados nas Tabelas 1 e 2.

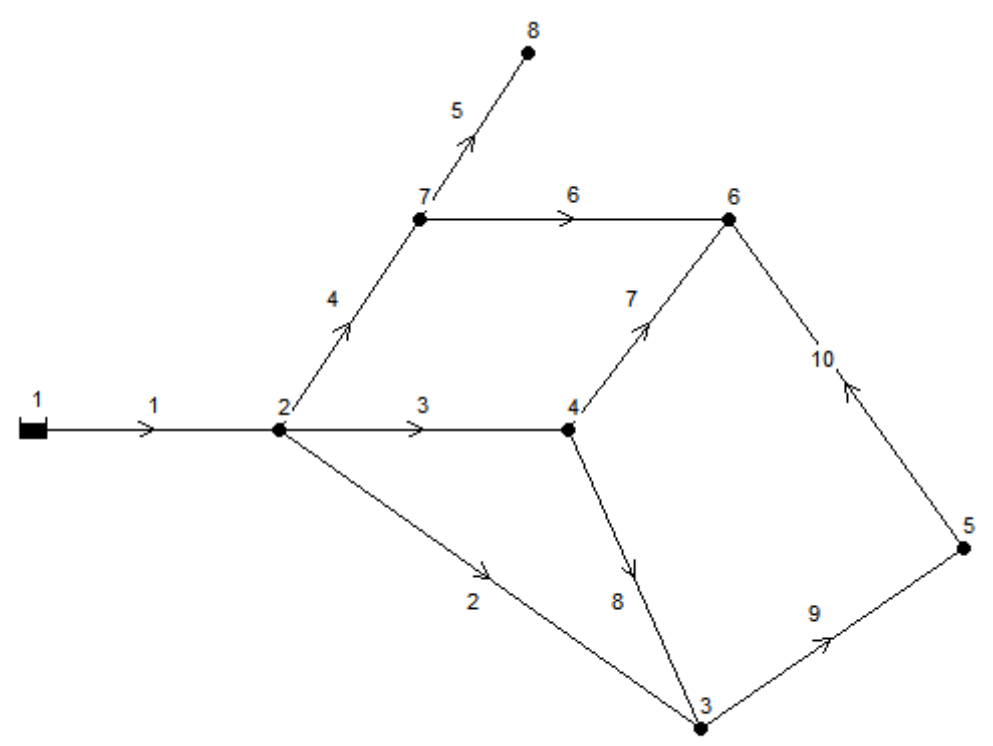

Figura 1 - Rede Walski (1983) and Gambale (2000) 
Tabela 1 - Dados referentes aos trechos - Rede Walski (1983) - Gambale (2000)

\begin{tabular}{|c|c|c|c|c|c|c|}
\hline \multirow{2}{*}{ Trecho } & \multirow{2}{*}{$\begin{array}{c}\text { Comprimento } \\
\text { (m) }\end{array}$} & \multirow{2}{*}{$\underset{(\mathrm{mm})}{D}$} & \multicolumn{2}{|c|}{ Rede Gabarito } & \multicolumn{2}{|c|}{ Rede Calculada } \\
\hline & & & $\varepsilon(\mathrm{mm})$ & Vazão (L/s) & $\varepsilon(\mathrm{mm})$ & Vazão (L/s) \\
\hline 1 & 700 & 500 & 0.0700 & 207.5000 & 0.0700 & 207.4996 \\
\hline 2 & 1800 & 250 & 0.0150 & 27.8107 & 0.0150 & 36.9975 \\
\hline 3 & 1520 & 400 & 0.0100 & 104.0722 & 0.0100 & 78.9518 \\
\hline 4 & 1220 & 300 & 0.0120 & 75.6172 & 0.0120 & 61.9074 \\
\hline 5 & 600 & 300 & 0.7000 & 37.5000 & 0.7000 & 29.6428 \\
\hline 6 & 1220 & 200 & 0.1000 & 8.1172 & 0.1000 & 2.6218 \\
\hline 7 & 920 & 250 & 0.0800 & 38.1139 & 0.0800 & 30.2507 \\
\hline 8 & 300 & 150 & 0.0600 & 3.4583 & 0.0600 & 19.0584 \\
\hline 9 & 600 & 200 & 0.9000 & 16.2690 & 0.9000 & 26.4131 \\
\hline 10 & 1220 & 100 & 1.0000 & 1.2690 & 1.0000 & -3.2297 \\
\hline
\end{tabular}

Tabela 2 - Dados referentes aos nós - Rede Walski (1983) - Gambale (2000)

\begin{tabular}{|c|c|c|c|c|c|}
\hline & \multicolumn{2}{|c|}{$\begin{array}{c}\text { Cota } \\
\text { Nó }\end{array}$} & \multicolumn{2}{|c|}{ Rede Gabarito } & \multicolumn{2}{|c|}{ Rede Calculada } \\
\hline & $\mathbf{( m )}$ & $\begin{array}{c}\text { Consumo } \\
\mathbf{( L / s )}\end{array}$ & $\begin{array}{c}\text { Pressão } \\
(\mathbf{m})\end{array}$ & $\begin{array}{c}\text { Consumo } \\
(\mathbf{L} / \mathbf{s})\end{array}$ & $\begin{array}{c}\text { Pressão } \\
(\mathbf{m})\end{array}$ \\
\hline $\mathbf{2}$ & 0 & 0 & 58.9454 & 29.65 & 58.9454 \\
\hline $\mathbf{3}$ & 0 & 15 & 56.9275 & 29.65 & 55.5513 \\
\hline $\mathbf{4}$ & 0 & 62.5 & 57.0240 & 29.65 & 57.7852 \\
\hline $\mathbf{5}$ & 0 & 15 & 55.6706 & 29.65 & 52.2847 \\
\hline $\mathbf{6}$ & 0 & 47.5 & 54.9876 & 29.65 & 56.4664 \\
\hline $\mathbf{7}$ & 0 & 30 & 55.4559 & 29.65 & 56.5276 \\
\hline $\mathbf{8}$ & 0 & 37.5 & 54.7211 & 29.65 & 56.0636 \\
\hline
\end{tabular}

\subsection{Rotina para Detecção de Possíveis Pontos de Vazamento}

Foram introduzidos pontos de vazamento nos trechos da rede gabarito em forma de nós, com as respectivas vazões como consumo nodal. A rede foi simulada utilizando a biblioteca do software EPANET2. Após a simulação, todas as pressões obtidas foram aplicadas nos respectivos nós da rede observada, desconsiderando os pontos de vazamento, em forma de reservatórios fictícios. A iteração foi realizada 100 vezes e adotou-se o menor valor da função objetivo representada pela Eq. 1.

Após o processo, observou-se que o consumo de cada vazamento inserido na rede se comportou de duas maneiras distintas. Quando o vazamento está localizado em um trecho, o valor do consumo é dividido entre os nós a montante e jusante do respectivo trecho. Se o vazamento for inserido apenas como um consumo adicional em qualquer nó da rede, este permanece no respectivo nó até o fim do processo iterativo utilizado para calibração.

Pela diferença entre o consumo da rede gabarito e o consumo calculado, observou-se que os nós que estão como condição de contorno dos trechos que foram inseridos os pontos com vazamento apresentaram um erro de consumo consideravelmente maior em relação aos outros nós da rede.

A rotina computacional proposta calcula os erros absolutos entre os consumos para todos os nós, ou seja, a diferença entre os consumos da rede gabarito e os consumos calculados por meio da calibração. Com os dados, a rotina analisa todos os nós que possuem erros entre os consumos maiores que um erro absoluto de 0,1 L/s, calculado pela Eq. 6. Se os nós selecionados forem identificados como condição de contorno (nós de montante e jusante) de algum trecho da rede analisada, este será identificado como um 
possível trecho com vazamento. Bezerra (2018) desenvolveu a Eq. 7 para identificar a posição do vazamento em um trecho.
A Fig. 2 representa o fluxograma da rotina computacional implementada para detecção de perdas de água em redes de abastecimento de água.

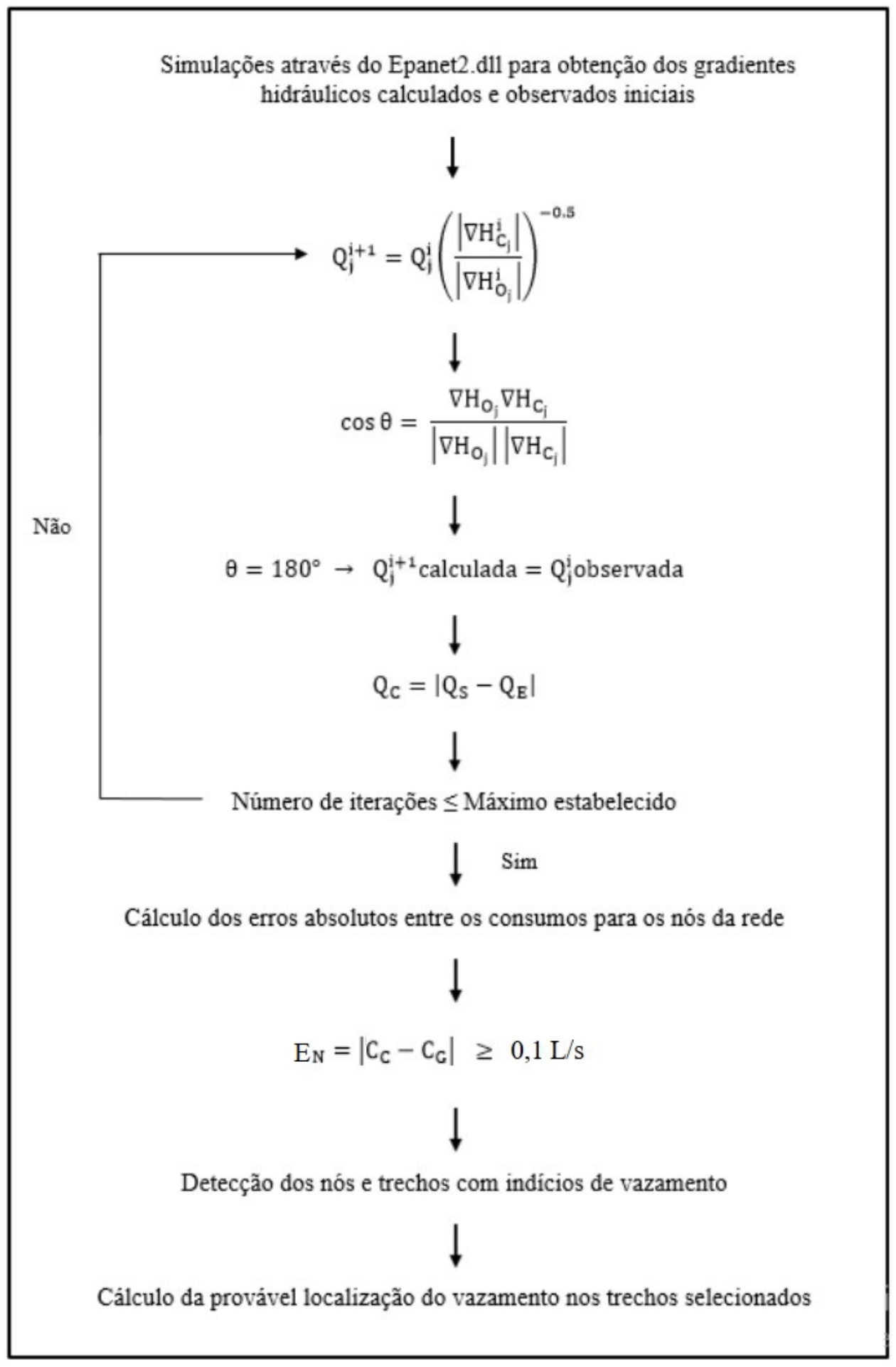

Figura 2 - Fluxograma da rotina proposta para a calibração da vazão, cálculo do consumo nodal e detecção de possíveis pontos de vazamento na rede 


$$
\mathrm{E}_{\mathrm{N}}=\left|C_{C}-C_{G}\right|
$$

Em que $E_{N}$ é o erro absoluto do nó, $C_{C}$ é o consumo calculado e $\mathrm{C}_{\mathrm{G}}$ é o consumo gabarito.

$\mathrm{D}_{\mathrm{V}}=\left\{1-\frac{\left(\mathrm{C} 1_{\mathrm{C}}-\mathrm{C} 1_{\mathrm{G}}\right)}{\left[\left(\mathrm{C} 1_{\mathrm{C}}-\mathrm{C} 1_{\mathrm{G}}\right)+\left(\mathrm{C} 2_{\mathrm{c}}-\mathrm{C} 2_{\mathrm{G}}\right)\right]}\right\} \mathrm{L}_{\mathrm{T}}$

Em que $D_{v}$ é a distância em metros do vazamento em relação ao nó 1 (pode ser tanto o nó de montante como o de jusante), $\mathrm{C}_{\mathrm{C}}$ é o consumo do nó 1 de um trecho calculado após a calibração, $\mathrm{C} 1_{G}$ é o consumo gabarito do nó 1 de um trecho, $\mathrm{C}_{\mathrm{c}}$ é o consumo do nó 2 de um trecho calculado após a calibração, $\mathrm{C} 2_{\mathrm{G}}$ é o consumo gabarito do nó 2 de um trecho e $L_{T}$ é o comprimento do trecho em metros. É importante perceber que o numerador da citada equação representa a perda encontrada no nó 1 e o denominador a perda total encontrada no trecho.

Em suma, a rotina desenvolvida utiliza o MIGHA para realizar a calibração das vazões nos trechos da rede de distribuição de água. De posse dos consumos da rede gabarito e os consumos calculados após a calibração, os erros absolutos entre estes são determinados para todos os nós pela Eq. 6. Se o erro calculado para um determinado nó for maior que $0,1 \mathrm{~L} / \mathrm{s}$, que é o resíduo máximo de vazão permitido segundo a NBR 12218/2017 (ABNT, 2017) em projetos de rede de distribuição de água para abastecimento público, a rotina identifica este como um possível nó com vazamento ou como condição de contorno (nó de montante ou jusante) de algum trecho com um possível vazamento. Se a rotina constatar que ambos os nós de montante e jusante de algum trecho da rede possuem os erros entre os consumos gabaritos e calculados maiores que o admissível, este é indicado como um trecho com indício de vazamento. A Eq. 7 é utilizada para calcular a provável posição do vazamento nos trechos identificados por meio da aplicação da rotina computacional desenvolvida.

\section{RESULTADOS E DISCUSSÕES}

As diferenças médias relativas a pressão, consumo e vazão após a utilização do método iterativo para a rede Rede Walski (1983) e Gambale (2000) estão listados na Tabela 3.

Tabela 3 - Erros médios calculados - Rede Walski (1983) - Gambale (2000)

\begin{tabular}{|c|c|c|c|}
\hline \multirow{2}{*}{$\begin{array}{c}\text { Nós com } \\
\text { pressões conhecidas }\end{array}$} & $\begin{array}{c}\text { Pressões } \\
\mathbf{( m )}\end{array}$ & $\begin{array}{c}\text { Consumo } \\
\text { nodal (L/s) }\end{array}$ & $\begin{array}{c}\text { Vazões } \\
\mathbf{( L / s )}\end{array}$ \\
\hline 2 & 1.3452 & 16.8361 & 11.1106 \\
\hline 2 e 3 & 1.0262 & 17.2224 & 9.0952 \\
\hline 2,3 e 4 & 0.7352 & 10.5731 & 4.7182 \\
\hline $2,3,4$ e 5 & 0.4418 & 7.4403 & 3.6642 \\
\hline $2,3,4,5$ e 6 & 0.1447 & 2.2445 & 1.5385 \\
\hline $2,3,4,5,6$ e 7 & 0.0388 & 2.2469 & 0.7872 \\
\hline $2,3,4,5,6,7$ e 8 & 0.0001 & 0.0020 & 0.0015 \\
\hline
\end{tabular}

Observou-se que quanto maior a quantidade de dados de pressão, menor o erro médio. Logo, a situação ideal para se fazer a análise das perdas de água na rede é a em que se possui dados de pressão em todos os nós, pois a diferença entre os consumos da rede gabarito e os consumos calculados retornam somente os nós que funcionam como condição de contorno dos trechos ponto de vazamento.

Vale ressaltar que é possível fazer a análise com menos dados de pressão, mas as chances de a rotina retornar nós ou trechos com uma detecção de vazamento falsa são consideravelmente maiores.

O primeiro teste foi realizado inserindo um nó para representar um vazamento no trecho $9 \mathrm{da}$ rede a 200 metros de distância do nó 3, com um consumo de $2 \mathrm{~L} / \mathrm{s}$, como mostra a Fig. 3. 


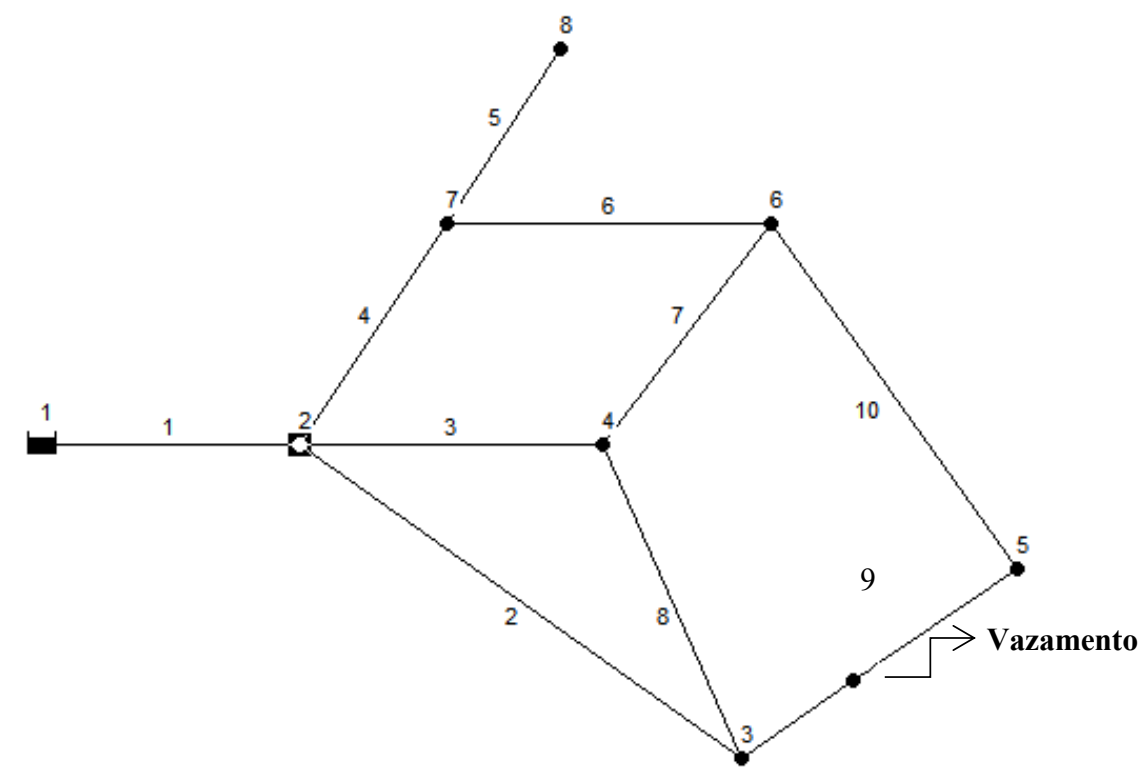

Figura 3 - Representação do vazamento inserido no trecho 9

A Tabela 4 mostra os erros entre os consumos por nó para diferentes quantidades de dados de pressão. Observa-se que, com todos os dados de pressão, apenas os nós que são condição de contorno do tre- cho 9 apresentaram um erro de consumo consideravelmente maior em relação aos outros nós da rede. Logo, para detectar os pontos de vazamento com maior precisão são necessárias todas as pressões.

Tabela 4 - Erros absolutos por nó para diferentes quantidades de dados de pressão (primeiro teste)

\begin{tabular}{|c|c|c|c|c|c|c|c|}
\hline \multirow{2}{*}{ Nó } & \multicolumn{7}{|c|}{ Quantidade de Dados de Pressão } \\
\hline & 1 & 2 & 3 & 4 & 5 & 6 & 7 \\
\hline 2 & 31.6455 & 46.3198 & 10.3304 & 10.7471 & 4.5092 & 0.0044 & 0.0044 \\
\hline 3 & 14.6428 & 0.2788 & 8.9932 & 1.2977 & 1.3033 & 1.3033 & 1.3033 \\
\hline 4 & 32.8572 & 32.6498 & 12.1715 & 13.0057 & 0.0001 & 0.0003 & 0.0003 \\
\hline 5 & 14.6428 & 14.6451 & 14.6436 & 2.9694 & 0.6949 & 0.6949 & 0.6949 \\
\hline 6 & 17.8572 & 17.8073 & 18.1515 & 17.9464 & 2.9977 & 0.0014 & 0.0014 \\
\hline 7 & 0.3572 & 0.3694 & 0.1412 & 0.2139 & 0.3546 & 7.8559 & 0.0010 \\
\hline 8 & 7.8572 & 7.8572 & 7.8572 & 7.8572 & 7.8572 & 7.8572 & 0.0002 \\
\hline
\end{tabular}

A rotina retornou que o trecho 9 da rede, assim como os nós 3 e 5, possuem indícios de vazamento. Utilizando a Eq. 6 para calcular a distância do vazamento em relação ao nó de montante, o resultado foi que o vazamento se encontra a 208,66 metros do nó 3 . O cálculo da distância para o primeiro teste foi satisfatório, apresentando um erro relativo de $4,15 \%$.
O segundo teste foi realizado inserindo um nó para representar um vazamento no trecho $9 \mathrm{da}$ rede a 200 metros de distância do nó 3, com um consumo de $2 \mathrm{~L} / \mathrm{s}$, e um nó no trecho 10 da rede a 610 metros de distância do nó 5 , com um consumo de $5 \mathrm{~L} / \mathrm{s}$, como mostra a Fig. 4. 


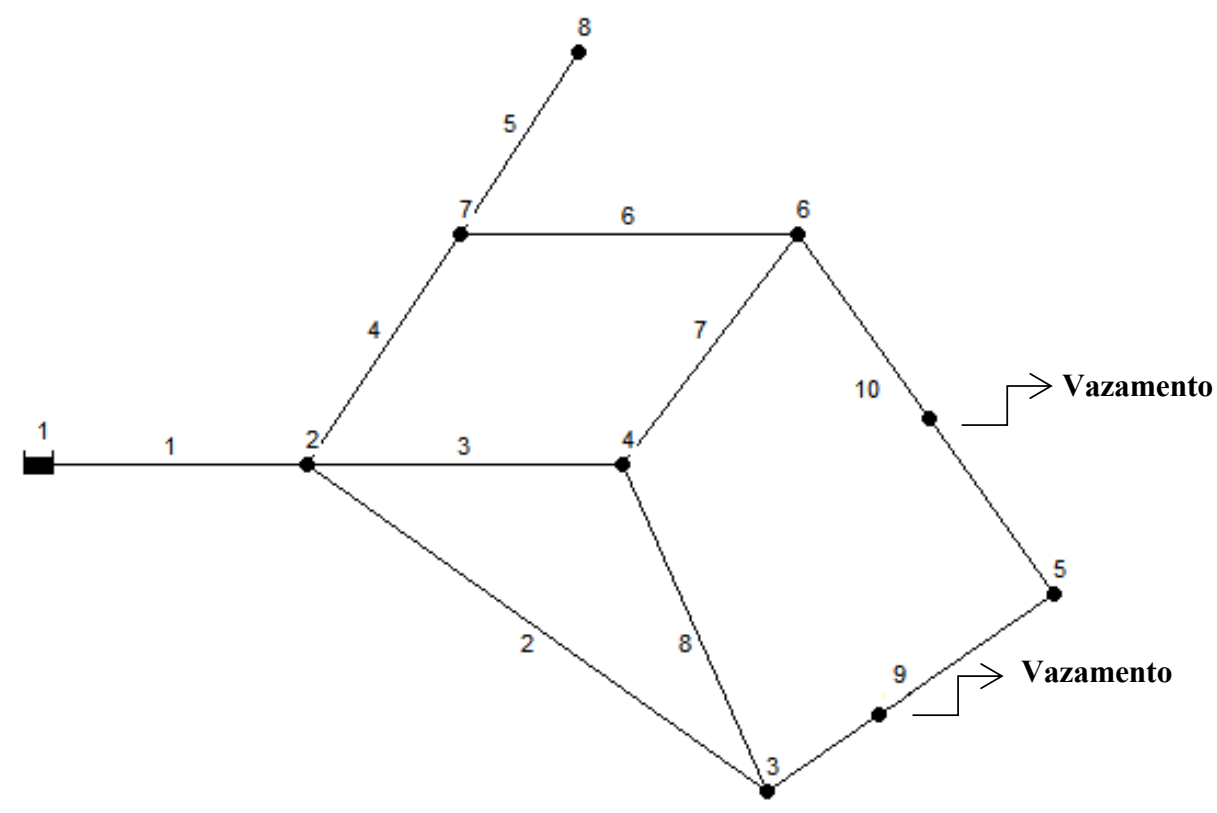

Figura 4 - Representação dos vazamentos inseridos nos trechos 9 e 10

A Tabela 5 mostra os erros absolutos entre os consumos por nó para diferentes quantidades de dados de pressão gabarito para o segundo teste.

Tabela 5 - Erros absolutos por nó para diferentes quantidades de dados de pressão (segundo teste)

\begin{tabular}{|l|c|c|c|c|c|c|c|c|}
\hline \multirow{2}{*}{ Nó } & \multicolumn{7}{|c|}{ Quantidade de Dados de Pressão } \\
\hline $\mathbf{2}$ & $\mathbf{1}$ & $\mathbf{2}$ & $\mathbf{3}$ & $\mathbf{4}$ & $\mathbf{5}$ & $\mathbf{6}$ & $\mathbf{7}$ \\
\hline $\mathbf{3}$ & 36.6457 & 49.6121 & 11.3762 & 11.6927 & 4.6014 & 0.0038 & 0.0038 \\
\hline $\mathbf{4}$ & 14.6428 & 1.4550 & 7.3427 & 1.3093 & 1.3093 & 1.3092 & 1.3092 \\
\hline $\mathbf{5}$ & 32.8572 & 32.6760 & 14.5407 & 15.2298 & 0.0014 & 0.0012 & 0.0012 \\
\hline $\mathbf{6}$ & 14.6428 & 14.6464 & 14.6260 & 4.8675 & 2.4109 & 2.4109 & 2.4109 \\
\hline $\mathbf{7}$ & 17.8572 & 17.8051 & 18.2330 & 18.1017 & 6.0564 & 3.2785 & 3.2785 \\
\hline $\mathbf{8}$ & 0.3572 & 0.3724 & 0.1071 & 0.1375 & 0.4807 & 3.2785 & 0.0032 \\
\hline
\end{tabular}

A rotina retornou que os trechos 9 e 10, assim como os nós 3, 5 e 6 possuem indícios de vazamento. Utilizando a Eq. 6 para calcular a distância dos vazamentos em relação aos nós de montante, o resultado foi que o vazamento no trecho 9 encontra-se a 388,84 metros do nó 3 e o vazamento no trecho 10 encontra-se a 703,02 metros do nó 5 . Os cálculos das distâncias nesse caso não foram satisfatórios, apresentando erros de $48,56 \%$ e $13,23 \%$, respectivamente. Após a calibração, parte dos consumos dos va- zamentos em ambos os trechos migraram para o nó 5, fazendo que o vazamento de um trecho interferisse na identificação do ponto de vazamento do outro.

O terceiro teste foi realizado inserindo um nó para representar um vazamento no trecho $7 \mathrm{da}$ rede a 460 metros de distância do nó 4 , com um consumo de $4 \mathrm{~L} / \mathrm{s}$, e um vazamento em forma de consumo adicional no nó 8 da rede, com um consumo de 2,5 L/s, como mostra a Fig. 5 . 


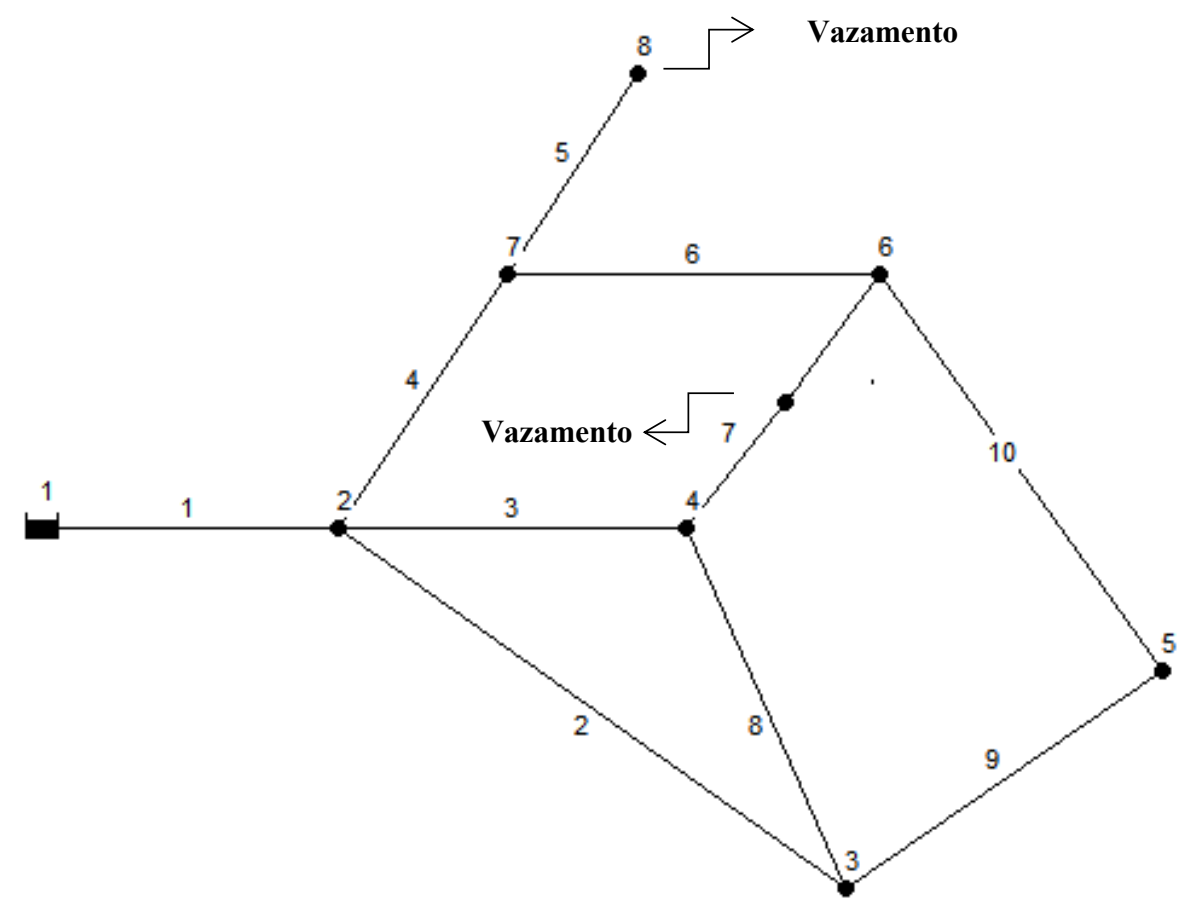

Figura 5 - Representação dos vazamentos inseridos no trecho 7 e nó 8

A Tabela 6 mostra os erros absolutos entre os consumos por nó para diferentes quantidades de dados de pressão gabarito para o terceiro teste.

Tabela 6 - Erros absolutos por nó para diferentes quantidades de dados de pressão (terceiro teste)

\begin{tabular}{|c|c|c|c|c|c|c|c|c|}
\hline \multirow{2}{*}{ Nó } & \multicolumn{7}{|c|}{ Quantidade de Dados de Pressão } \\
\hline & $\mathbf{1}$ & $\mathbf{2}$ & $\mathbf{3}$ & $\mathbf{4}$ & $\mathbf{5}$ & $\mathbf{6}$ & $\mathbf{7}$ \\
\hline $\mathbf{2}$ & 36.1450 & 51.0276 & 12.6364 & 13.0760 & 6.0125 & 0.0035 & 0.0035 \\
\hline $\mathbf{3}$ & 14.6428 & 0.4907 & 10.7187 & 0.0001 & 0.0018 & 0.0018 & 0.0018 \\
\hline $\mathbf{4}$ & 32.8572 & 32.6464 & 16.0805 & 17.0327 & 1.9505 & 1.9502 & 1.9502 \\
\hline $\mathbf{5}$ & 14.6428 & 14.6450 & 14.6494 & 2.4465 & 0.0001 & 0.0001 & 0.0001 \\
\hline $\mathbf{6}$ & 17.8572 & 17.8071 & 18.1694 & 18.0368 & 5.9366 & 2.0459 & 2.0459 \\
\hline $\mathbf{7}$ & 0.3572 & 0.3692 & 0.1189 & 0.1591 & 0.4577 & 10.3578 & 0.0011 \\
\hline $\mathbf{8}$ & 7.8572 & 7.8572 & 7.8572 & 7.8572 & 7.8572 & 7.8572 & 2.4995 \\
\hline
\end{tabular}

A rotina retornou que o trecho 7 , assim como os nós 4, 6 e 8 da rede possuem indícios de vazamentos. Utilizando a Eq. 6 para calcular a distância dos vazamentos em relação ao nó de montante, o resultado foi que o vazamento no trecho 7 encontra-se 471,01 metros do nó 4 . O cálculo da distância nesse teste foi satisfatório, apresentando um erro relativo de $2,33 \%$.

O quarto teste realizado foi semelhante ao terceiro, alterando somente o vazamento do nó 8 para o 6, como mostra a Fig. 6. 


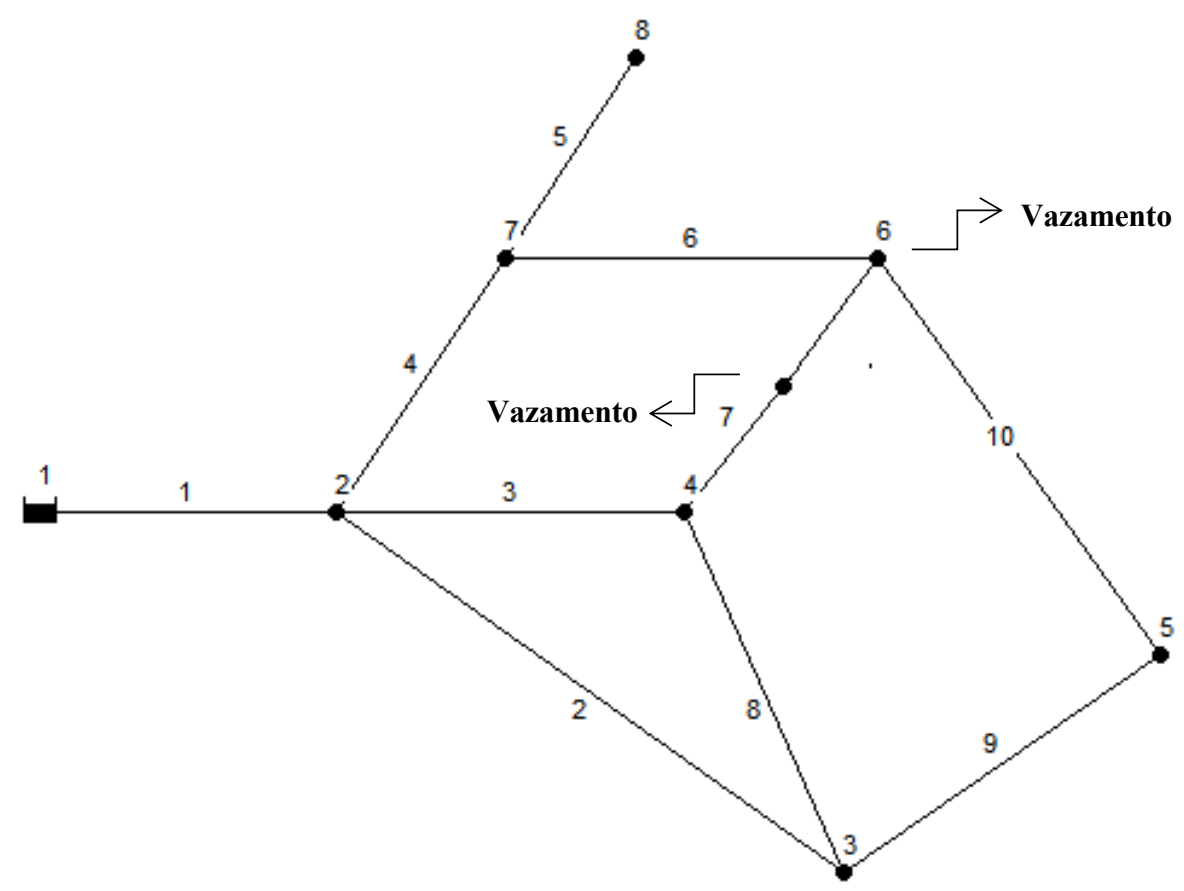

Figura 6 - Representação dos vazamentos inseridos no trecho 7 e nó 6

A Tabela 7 mostra os erros absolutos entre os consumos por nó para diferentes quantidades de dados de pressão gabarito para o quarto teste.

Tabela 7 - Erros absolutos por nó para diferentes quantidades de dados de pressão (quarto teste)

\begin{tabular}{|l|l|l|l|l|l|l|l|}
\hline \multirow{2}{*}{ Nó } & \multicolumn{7}{|c|}{ Quantidade de Dados de Pressão } \\
\hline $\mathbf{2}$ & $\mathbf{1}$ & $\mathbf{2}$ & $\mathbf{3}$ & $\mathbf{4}$ & $\mathbf{5}$ & $\mathbf{6}$ & $\mathbf{7}$ \\
\hline $\mathbf{3}$ & 35.1404 & 49.9386 & 11.3311 & 11.7683 & 4.5899 & 0.0023 & 0.0023 \\
\hline $\mathbf{4}$ & 14.6428 & 0.4047 & 10.6977 & 0.0002 & 0.0021 & 0.0021 & 0.0021 \\
\hline $\mathbf{5}$ & 32.8572 & 32.6478 & 16.3613 & 17.3116 & 1.9521 & 1.9519 & 1.9519 \\
\hline $\mathbf{6}$ & 14.6428 & 14.6450 & 14.6494 & 2.4716 & 0.0003 & 0.0003 & 0.0003 \\
\hline $\mathbf{7}$ & 17.8572 & 17.8072 & 18.1732 & 18.0408 & 6.3390 & 3.5469 & 3.5469 \\
\hline $\mathbf{8}$ & 0.3572 & 0.3693 & 0.1162 & 0.1562 & 0.4719 & 7.8568 & 0.0002 \\
\hline
\end{tabular}

A rotina retornou que o trecho 7 e os nós 4 e 6 possuem indícios de vazamento. Utilizando a Eq. 6 para calcular a distância do vazamento em relação ao nó de montante, o resultado foi que o provável vazamento no trecho 7 encontra-se a 593.43 metros do nó 4 . 0 cálculo da distância nesse caso não foi satisfatório, apresentando um erro relativo de $26,26 \%$. O consumo adicional atribuído ao nó 6 interferiu no cálculo da distância do vazamento do trecho comum a este mesmo nó.

\section{CONCLUSÃO}

Para se obterem resultados mais precisos, é necessário ter dados de pressão para todos os nós da rede analisada, pois a diferença média após a calibração com todos os dados de pressão teve resultados muito melhores do que todas as outras simulações com menor quantidade de dados.

A rotina computacional implementada para detecção de possíveis vazamentos na rede obteve 
resultados satisfatórios para os testes em que os pontos de vazamento estavam inseridos em trechos isolados de qualquer outro vazamento, ou seja, primeiro e terceiro teste. Nessa situação, a Eq. 6 conseguiu estimar aproximadamente o ponto em que o vazamento está localizado, pois os consumos dos nós de montante e jusante deste trecho não sofreram qualquer influência de outro vazamento.

Para o segundo e o quarto teste, a Eq. 6 não conseguiu estimar de forma satisfatória a localização dos pontos com vazamento, visto que os nós de montante e jusante sofreram influência de vazamentos em trechos ou nós adjacentes.

A rotina não conseguiu determinar com exatidão os vazamentos localizados nos nós da rede. Ela retorna todos os nós com os erros entres os consumos maiores que o erro permitido como possíveis pontos com vazamento, e os trechos que compartilham esses nós, como possíveis trechos com vazamento.

A metodologia apresentada objetiva consolidarse, principalmente, em relação à quantidade de dados observados de pressão necessária para realizar a calibração das vazões nos trechos das redes, mas ainda assim possibilita uma visão mais detalhada do emprego de métodos computacionais para detecção de perdas de água por meio da engenharia reversa. A validação da rotina proposta pode ser obtida a partir da utilização de métodos acústicos no intuito de verificar os resultados da aplicação do modelo.

Para redes em geral, mesmo que a rotina não consiga, em algumas situações, estimar os pontos exatos onde se encontram os vazamentos, ela ainda consegue localizar os possíveis nós e trechos em que os vazamentos estão localizados.

\section{CONTRIBUIÇÃO DOS AUTORES}

Todos os autores contribuíram de forma igualitária.

\section{REFERÊNCIAS}

ASSOCIAÇÃO BRASILEIRA DE NORMAS TÉCNICAS. NBR 12.218 Projeto de rede de distribuição de água para abastecimento público - Procedimento. Rio de Janeiro: ABNT, 2017.

BEZERRA, A. A.; CASTRO, M. A. H.; ARAUJO, R. S. A. Absolute roughness calculation by the friction factor calibration using the Alternative Hydraulic Gradient Iterative Method on water distribution networks. Revista Brasileira de Recursos Hídricos, v.22, e24, 2017. https://doi.org/10.1590/2318 - 0331.021720160018

BEZERRA, A. A. Modelagem numérica computacional aplicada à calibração através de novas equações para o uso do método iterativo do gradiente hidráulico/concentração alternativo (MIGHA) em redes de distribuição de água. Tese (Doutorado em Recursos Hídricos) - Universidade Federal do Ceará, Fortaleza, 2018.

GAMBALE, S. R. Aplicação de algoritmo genético na calibração de rede de água. 2000.266 f. Dissertação (Mestrado em Recursos Hídricos) - Escola Politécnica, Universidade de São Paulo, São Paulo, 2000.

TSUTIYA, M. T. Abastecimento de Água. 3. ed., São Paulo: Departamento de Engenharia Hidráulica e Sanitária da Escola Politécnica da Universidade de São Paulo, 2006.

SOARES, A. K.; REIS, L. F. R. Calibração de Modelos de Redes de Distribuição de Água Utilizando Modelo de Simulação Hidráulica Dirigido pela Pressão (MSHDP) e Método Híbrido AG-Simplex. Revista Brasileira de Recursos Hídricos. v. 9, n. 2, p. 85-96, 2004.

SISTEMA NACIONAL DE INFORMAÇÕES SOBRE SANEAMENTO. Diagnóstico dos serviços de água e esgotos - 2015. Brasília, 2017.

SCHUSTER, H. D. M.; ARAÚJO, H. D. B. Uma Formulação Alternativa do Método Iterativo de Gradiente Hidráulico no Procedimento de Calibração dos Parâmetros 316 Hidrodinâmicos do Sistema Aquífero. Revista Brasileira de Recursos Hídricos. v. 9, n 2, p. 31-37, 2004.

ROCHA, V. A. G. M.; CASTRO, M. A. H.; ARAÚJO, J. K. Calibração de Rugosidade em Redes de Abastecimento a Partir de Gradientes Hidráulicos Através de Método Iterativo. In: XVIII Simpósio Brasileiro de Recursos Hídricos. 2009. Anais...

WALSKI, T. M. Technique for Calibrating Network Models. Journal of Water Resources Planning and Management. v. 109, n. 4, p. 360-372, 1983 